\title{
Fluid Motion in the Earth's Core Derived from the Geomagnetic Field and Its Implications for the Geodynamo
}

\author{
Masaki Matsushima \\ Department of Earth and Planetary Sciences, Tokyo Institute of Technology, \\ Ookayama 2-12-1, Meguro-ku, Tokyo 152, Japan
}

(Received November 11, 1992; Revised August 1, 1993; Accepted August 28, 1993)

\begin{abstract}
An attempt is made to derive fluid motion in the Earth's outer core from geomagnetic field data. We implicitly incorporate the energy source for the geodynamo in the prescribed radial dependence of poloidal velocity field. Then the Navier-Stokes equation for the toroidal constituent and the induction equation for the toroidal and the poloidal magnetic fields are solved so as to fit the magnetic field at the core-mantle boundary estimated through downward continuation on the assumption that the mantle is an insulator. The basic standpoint is that the large-scale magnetic field is maintained by induction processes associated with large-scale fluid motion within the core. We also impose the condition that time variations of the velocity and the magnetic fields are very slow. The generation balance in the induction equation, for the derived velocity and magnetic fields, suggests that the geodynamo is likely to be of $\alpha^{2} \omega$-type.
\end{abstract}

\section{Introduction}

For understanding of a realistic dynamo mechanism, it is essential to clarify fluid motion within the core which generates the Earth's magnetic field. Based on the frozen-flux approximation (ROBERTS and SCOTT, 1965), fluid motions near the core surface have been derived by many authors (WhaleR, 1980; GubBins, 1982; Le MouËL et al., 1985; VoORHIES, 1986; Whaler and Clarke, 1988; Bloxham, 1989; Lloyd and Gubbins, 1990; JaCkson and Bloxham, 1991) with additional constraints required to avoid the fundamental non-uniqueness (BACKUS, 1968). These studies are reviewed by BLOXHAM and JACKSON (1991).

On the other hand, the estimation of fluid motion near the core surface was attempted on the presumption that a strong zonal toroidal magnetic field is generated by the $\omega$-effect (RIKITAKE, 1967; Honkura and Rikitake, 1972; Honkura and MATSUShima, 1988). It was simply assumed that the non-axisymmetric poloidal magnetic fields are generated simply by the interaction between non-axisymmetric poloidal velocity fields and such a strong zonal toroidal magnetic field. MATSUSHIMA and HoNkURA (1989) improved the method by estimating a differential rotation, which is expected to generate a strong zonal toroidal magnetic field, and estimated fluid motion within the core. In their estimation of the differential rotation, however, they relied on virtual displacement of fluid particles, which is a physically too simplified model. MATsushima and HONKURA (1992) further improved the method and attempted to derive fluid motion within the core by solving both the Navier-Stokes and the induction equations, again on the presumption that zonal toroidal motion generates strong zonal toroidal magnetic fields which serve as the inducing magnetic fields for non-axisymmetric poloidal magnetic fields. They pointed out that no strong zonal toroidal magnetic field arises and thus the presumption fails; that is, we should not rely on any approximations for dominant induction terms, such as the $\omega$-effect.

In this paper, we attempt to include all the interaction terms and derive fluid motion within the core by solving the Navier-Stokes and the induction equations. We prescribe the radial dependence of poloidal velocity field, in which the energy source for the dynamo is implicitly 
incorporated, and assume that time variations of the velocity and the magnetic fields are very slow. In this sense, as mentioned in MATsushima and Honkura (1992), the present approach is regarded as an intermediate one between kinematic and magnetohydrodynamic (MHD) dynamos; that is, the velocity field is not completely given as in kinematic dynamo approaches, but determined so as to maintain the magnetic field as observed at the Earth's surface by taking the dynamics within the core into account.

Special emphasis is put on which type of mechanism is relevant to the geodynamo, and we investigate the generation balance in the induction equation using the derived velocity and magnetic fields.

\section{Method}

Mathematical formulation in this paper is nearly the same as in MATSUSHIMA and HoNKURA (1992). We solve the induction equation

$$
\frac{\partial \boldsymbol{B}}{\partial t}=\nabla \times(\boldsymbol{V} \times \boldsymbol{B})+\frac{1}{\mu_{0} \sigma} \nabla^{2} \boldsymbol{B},
$$

and the Navier-Stokes equation in the Boussinesq approximation

$$
\frac{\partial \boldsymbol{V}}{\partial t}+(\boldsymbol{V} \cdot \nabla) \boldsymbol{V}=-\nabla \varpi-2 \boldsymbol{\Omega} \times \boldsymbol{V}+\frac{1}{\rho \mu_{0}}(\nabla \times \boldsymbol{B}) \times \boldsymbol{B}+C \boldsymbol{g}+\nu \nabla^{2} \boldsymbol{V}
$$

for the toroidal constituent. In these equations, $\boldsymbol{B}$ is the magnetic field, $\boldsymbol{V}$ the velocity field, $\mu_{0}$ the magnetic permeability of vacuum, $\sigma$ the electrical conductivity of the core, $\varpi$ the reduced pressure, $\boldsymbol{\Omega}$ the angular velocity of the mantle, $\rho$ the mean density of the core, $C$ the buoyancy parameter, $\boldsymbol{g}$ the gravitational acceleration vector, and $\nu$ the kinematic viscosity of core fluid.

The velocity field $\boldsymbol{V}$ and the magnetic field $\boldsymbol{B}$ can be expressed in terms of toroidal and poloidal vectors, since they are divergence-free;

$$
\begin{aligned}
\boldsymbol{V}(\boldsymbol{r}, t) & =\boldsymbol{V}_{T}(\boldsymbol{r}, t)+\boldsymbol{V}_{S}(\boldsymbol{r}, t) \\
& =\nabla \times(W(\boldsymbol{r}, t) \hat{\boldsymbol{r}})+\nabla \times \nabla \times(U(\boldsymbol{r}, t) \hat{\boldsymbol{r}})
\end{aligned}
$$

and

$$
\begin{aligned}
\boldsymbol{B}(\boldsymbol{r}, t) & =\boldsymbol{B}_{T}(\boldsymbol{r}, t)+\boldsymbol{B}_{S}(\boldsymbol{r}, t) \\
& =\nabla \times(T(\boldsymbol{r}, t) \hat{\boldsymbol{r}})+\nabla \times \nabla \times(S(\boldsymbol{r}, t) \hat{\boldsymbol{r}})
\end{aligned}
$$

where $\boldsymbol{r}$ is the position vector, $\hat{\boldsymbol{r}}$ the radial unit vector, and $t$ the time. $W, U, T$ and $S$ are scalar functions for toroidal velocity field $\boldsymbol{V}_{T}$, poloidal velocity field $\boldsymbol{V}_{S}$, toroidal magnetic field $\boldsymbol{B}_{T}$, and poloidal magnetic field $\boldsymbol{B}_{S}$, respectively.

We consider large-scale velocity and large-scale magnetic fields. Scalar functions for $\boldsymbol{V}$ and $\boldsymbol{B}$ are then expanded into a series of spherical harmonics in the spherical coordinates $(r, \theta, \phi)$;

$$
\begin{aligned}
W(\boldsymbol{r}, t) & =\sum_{l=1}^{L} \sum_{m=0}^{l}\left\{W_{l}^{m c}(r, t) \cos m \phi+W_{l}^{m s}(r, t) \sin m \phi\right\} P_{l}^{m}(\cos \theta), \\
U(\boldsymbol{r}, t) & =\sum_{l=1}^{L} \sum_{m=0}^{l}\left\{U_{l}^{m c}(r, t) \cos m \phi+U_{l}^{m s}(r, t) \sin m \phi\right\} P_{l}^{m}(\cos \theta),
\end{aligned}
$$




$$
T(\boldsymbol{r}, t)=\sum_{l=1}^{L} \sum_{m=0}^{l}\left\{T_{l}^{m c}(r, t) \cos m \phi+T_{l}^{m s}(r, t) \sin m \phi\right\} P_{l}^{m}(\cos \theta)
$$

and

$$
S(\boldsymbol{r}, t)=\sum_{l=1}^{L} \sum_{m=0}^{l}\left\{S_{l}^{m c}(r, t) \cos m \phi+S_{l}^{m s}(r, t) \sin m \phi\right\} P_{l}^{m}(\cos \theta),
$$

where $P_{l}^{m}(\cos \theta)$ is an associated Legendre function of degree $l$ and order $m$, and $L$ the truncation level in the degree of spherical harmonics.

Following BULlaRD and GELlmaN (1954), the induction equation is given as

$$
\begin{aligned}
r^{2} \frac{\partial T_{\gamma}}{\partial t} & =\frac{1}{\mu_{0} \sigma}\left\{r^{2} \frac{\partial^{2} T_{\gamma}}{\partial r^{2}}-\gamma(\gamma+1) T_{\gamma}\right\} \\
& +\sum_{\alpha} \sum_{\beta}\left\{\left(U_{\alpha} S_{\beta} t_{\gamma}\right)+\left(W_{\alpha} S_{\beta} t_{\gamma}\right)+\left(U_{\alpha} T_{\beta} t_{\gamma}\right)+\left(W_{\alpha} T_{\beta} t_{\gamma}\right)\right\} \\
r^{2} \frac{\partial S_{\gamma}}{\partial t} & =\frac{1}{\mu_{0} \sigma}\left\{r^{2} \frac{\partial^{2} S_{\gamma}}{\partial r^{2}}-\gamma(\gamma+1) S_{\gamma}\right\} \\
& +\sum_{\alpha} \sum_{\beta}\left\{\left(U_{\alpha} S_{\beta} s_{\gamma}\right)+\left(W_{\alpha} S_{\beta} s_{\gamma}\right)+\left(U_{\alpha} T_{\beta} s_{\gamma}\right)\right\}
\end{aligned}
$$

In the same way, following FrAzER (1974), the Navier-Stokes equation for the toroidal constituent is given as

$$
\begin{aligned}
r^{2} \frac{\partial W_{\gamma}}{\partial t}= & \nu\left\{r^{2} \frac{\partial^{2} W_{\gamma}}{\partial r^{2}}-\gamma(\gamma+1) W_{\gamma}\right\}-2 \Omega \sum_{\alpha}\left\{\left(U_{\alpha} S_{1}^{\Omega} s_{\gamma}\right)+\left(W_{\alpha} S_{1}^{\Omega} s_{\gamma}\right)\right\} \\
& +\frac{1}{\rho \mu_{0}} \sum_{\alpha} \sum_{\beta}\left\{\left[S_{\alpha} S_{\beta} s_{\gamma}\right]+\left[T_{\alpha} S_{\beta} s_{\gamma}\right]+\left[T_{\alpha} T_{\beta} s_{\gamma}\right]\right\} \\
& -\sum_{\alpha} \sum_{\beta}\left\{\left[U_{\alpha} U_{\beta} s_{\gamma}\right]+\left[W_{\alpha} U_{\beta} s_{\gamma}\right]+\left[W_{\alpha} W_{\beta} s_{\gamma}\right]\right\}
\end{aligned}
$$

In these equations, Greek letters, $\alpha, \beta$ and $\gamma$, appearing as a subscript denote the set of $(l, m, z)$, where $z=c$ or $z=s$ according to the cosine or sine term, and those appearing otherwise denote the degree $l$ of the spherical harmonics, as in Bullard and GELlman (1954). The induction and Coriolis force terms in parentheses are given by Bullard and Gellman (1954), and the Lorentz force and advection terms in brackets by FrAZER (1974). In these interaction terms, $s_{\gamma}$ and $t_{\gamma}$ denote radial functions of poloidal and toroidal vectors, respectively, which are equal to unity for all $r$, and are introduced to obtain (6), (7) and (8), using the orthogonality of spherical harmonics. The scalar function $S_{1}^{\Omega}(r)$, which arises from the angular velocity $\Omega$, is given as $r^{2} / 2$.

In this paper, we impose the stress-free boundary conditions at the inner core boundary (ICB) and the core-mantle boundary (CMB), because the Ekman number $E$ is of the order of $10^{-9}$ even for the kinematic viscosity $\nu=1 \mathrm{~m}^{2} / \mathrm{s}$, as adopted by MATSUSHIMA and HonkURA (1992), and the Ekman layer cannot simply be resolved. We then have the boundary conditions for the velocity field as

$$
U_{\alpha}=\frac{\partial^{2}}{\partial r^{2}} \frac{U_{\alpha}}{r}=\frac{\partial}{\partial r} \frac{W_{\alpha}}{r^{2}}=0 .
$$

We assume that the mantle is an electrical insulator. As for the inner core, we consider two extreme cases. One is that the inner core is also assumed to be an insulator $\left(\sigma_{i c}=0\right)$. This 
case is not realistic, but drastic changes of results are not expected even if the realistic boundary conditions were imposed because of the very small volume occupied by the inner core (ZHANG and Busse, 1988, 1989).

In this case, the toroidal magnetic field vanishes outside the outer core, and the poloidal magnetic field must be matched to a potential magnetic field at both the boundaries. The boundary conditions for the magnetic field are then given as

$$
\begin{aligned}
T_{\beta}=r \frac{\partial S_{\beta}}{\partial r}+\beta S_{\beta}=0 & \text { at } r=r_{o c}, \\
T_{\beta}=r \frac{\partial S_{\beta}}{\partial r}-(\beta+1) S_{\beta}=0 & \text { at } r=r_{i c},
\end{aligned}
$$

where $r_{o c}$ and $r_{i c}$ denote the radii of the outer and the inner cores, respectively.

The other case is that the inner core is assumed to be a perfect conductor. Then the radial component of the magnetic field and the horizontal component of the electric field must vanish at the ICB. The boundary conditions for the magnetic field in the case of $\sigma_{i c}=\infty$ are given as

$$
\begin{array}{ll}
T_{\beta}=r \frac{\partial S_{\beta}}{\partial r}+\beta S_{\beta}=0 & \text { at } r=r_{o c} \\
\frac{\partial T_{\beta}}{\partial r}=S_{\beta}=\frac{\partial^{2} S_{\beta}}{\partial r^{2}}=0 & \text { at } r=r_{i c} .
\end{array}
$$

However, only the first and the third are required in (11b) since the second vanishes at the ICB whenever the radial components of the velocity and the magnetic fields vanish, as verified from the radial component of the induction equation (GLATZMAIER, 1984).

Scalar functions $U, W, S$ and $T$ are expanded into complete systems of functions satisfying the boundary conditions (9), and (10) or (11) (e.g. ZHANG and Busse, 1988)

$$
\begin{aligned}
U_{l}^{m}(r, t)= & \sum_{n=1}^{N}{ }_{n} U_{l}^{m}(t) r \sin \left(n \pi \frac{r-r_{i c}}{r_{o c}-r_{i c}}\right), \\
W_{l}^{m}(r, t)=\sum_{n=1}^{N}{ }_{n} W_{l}^{m}(t) r^{2} \cos \left((n-1) \pi \frac{r-r_{i c}}{r_{o c}-r_{i c}}\right), & \\
S_{l}^{m}(r, t) & =\sum_{n=1}^{N}{ }_{n} S_{l}^{m}(t) \cos \left({ }_{n} \alpha_{l} r-{ }_{n} \beta_{l}\right), \\
T_{l}^{m}(r, t) & = \begin{cases}\sum_{n=1}^{N}{ }_{n} T_{l}^{m}(t) r \sin \left(n \pi \frac{r-r_{i c}}{r_{o c}-r_{i c}}\right) & \text { for } \sigma_{i c}=0 \\
\sum_{n=1}^{N}{ }_{n} T_{l}^{m}(t) \sin \left(\frac{(2 n-1) \pi}{2} \frac{r+r_{o c}-2 r_{i c}}{r_{o c}-r_{i c}}\right) & \text { for } \sigma_{i c}=\infty\end{cases}
\end{aligned}
$$

where $N$ is the truncation level for radial functions. The constants ${ }_{n} \alpha_{l}$ and ${ }_{n} \beta_{l}$ are obtained by solving the algebraic equations corresponding to the boundary conditions (10) or (11). In the case of $\sigma_{i c}=0$,

$$
\begin{aligned}
{ }_{n} \alpha_{l} r_{o c} \tan \left({ }_{n} \alpha_{l} r_{o c}-{ }_{n} \beta_{l}\right) & =l, \\
{ }_{n} \alpha_{l} r_{i c} \tan \left({ }_{n} \alpha_{l} r_{i c}-{ }_{n} \beta_{l}\right) & =-(l+1),
\end{aligned}
$$

whereas in the case of $\sigma_{i c}=\infty$,

$$
\begin{aligned}
{ }_{n} \alpha_{l} r_{o c} \tan \left({ }_{n} \alpha_{l} r_{o c}-{ }_{n} \beta_{l}\right) & =l, \\
\cos \left({ }_{n} \alpha_{l} r_{i c}-{ }_{n} \beta_{l}\right) & =0 .
\end{aligned}
$$


Table 1. Coefficients of the expansion of scalar function for the poloidal velocity field.

\begin{tabular}{rr}
\hline$n$ & \multicolumn{1}{c}{${ }_{n} U_{l}^{m}$} \\
\hline 1 & $1.9844796159713667 \times 10^{+0}$ \\
2 & $-1.1208241951419211 \times 10^{+0}$ \\
3 & $1.6751870950105818 \times 10^{-1}$ \\
4 & $-5.0778639698902950 \times 10^{-2}$ \\
5 & $1.1877877699228212 \times 10^{-2}$ \\
6 & $-8.0662971235696546 \times 10^{-3}$ \\
7 & $1.6422307232510647 \times 10^{-3}$ \\
8 & $-2.3077201430485861 \times 10^{-3}$ \\
9 & $2.3799911429093004 \times 10^{-4}$ \\
10 & $-9.1618034466525028 \times 10^{-4}$ \\
\hline
\end{tabular}

These values were determined so as to fit the radial dependence of poloidal velocity field adopted in MATSUSHIMA and HONKURA (1992).

From (12c) and (14b), it is verified that the second and the third conditions in (11b) are satisfied.

As in MATSUSHIMA and HonkURA (1992), the radial dependence of poloidal velocity field is prescribed. The expansion of the scalar function for the poloidal velocity field is then rewritten as

$$
U_{l}^{m}(r, t)=V_{l}^{m}(t) \sum_{n=1}^{N}{ }_{n} U_{l}^{m} r \sin \left(n \pi \frac{r-r_{i c}}{r_{o c}-r_{i c}}\right),
$$

where ${ }_{n} U_{l}^{m}$ 's are prescribed and $V_{l}^{m}(t)$ 's are unknowns to be determined. We again adopt a similar radial dependence and simply assume that the radial function is independent of degree $l$ and order $m$ of spherical harmonics, as in MATsushima and HonkurA (1992), although the boundary condition at the ICB is different. The function with respect to $r$ is shown in Fig. 1 and ${ }_{n} U_{l}^{m}$ 's are given in Table 1.

Substituting (12) into (6), (7) and (8), we obtain the non-linear simultaneous equations. In addition, the poloidal magnetic field to be derived must fit the magnetic field at the CMB estimated through downward continuation on the assumption that the mantle is an insulator, that is

$$
\frac{l(l+1)}{r_{o c}^{2}} S_{l}^{m c}\left(r_{o c}, t\right)=(l+1)\left(\frac{r_{e}}{r_{o c}}\right)^{l+2} g_{l}^{m}(t)
$$

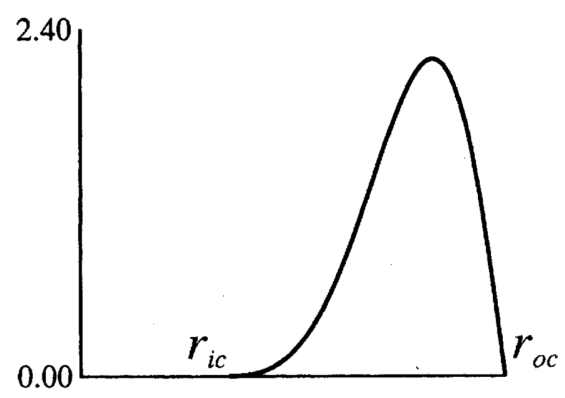

Fig. 1. Radial dependence of scalar function for the poloidal velocity field. 


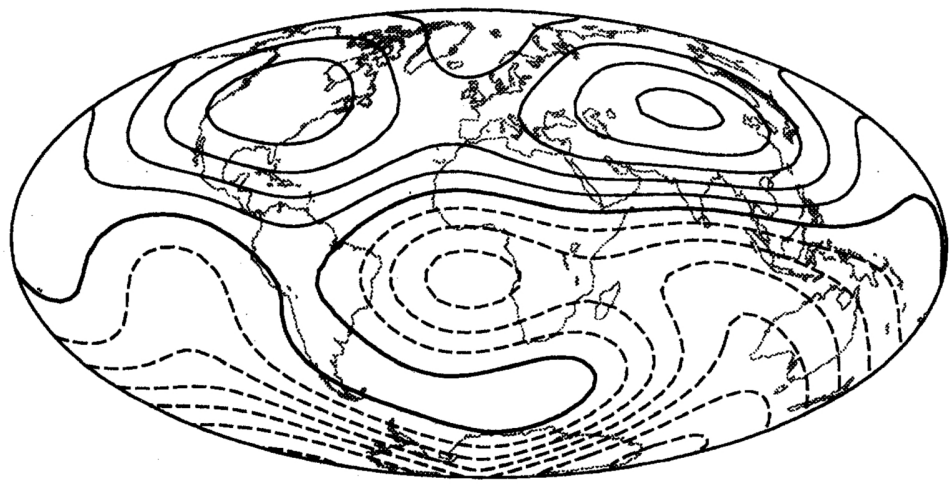

Fig. 2. The distribution of the radial component of the magnetic field, synthesized from spherical harmonics of degree up to four, at the CMB for the epoch of 1980 AD. The DGRF 1980 is used as the magnetic field data. Contour intervals are $100 \mu \mathrm{T}$. Solid contours represent magnetic flux into the core and broken contours magnetic flux out of the core. Bold contours represent null radial field lines.

$$
\frac{l(l+1)}{r_{o c}^{2}} S_{l}^{m s}\left(r_{o c}, t\right)=(l+1)\left(\frac{r_{e}}{r_{o c}}\right)^{l+2} h_{l}^{m}(t),
$$

where $g_{l}^{m}$ 's and $h_{l}^{m}$ 's are the Gauss coefficients and $\dot{r}_{e}$ denotes the Earth's mean radius. We then add (16) to the simultaneous equations to be solved. For the Gauss coefficients in (16), we use the DGRF 1980 (IAGA, 1988). We show, in Fig. 2, the distribution of the radial component of the magnetic field, up to degree four in spherical harmonics used in this paper, at the CMB for the epoch of 1980 AD. Solid contours represent magnetic flux into the core and broken contours magnetic flux out of the core, in units of $100 \mu \mathrm{T}$.

As in MATSUshima and HonkURA (1992), we assume the steady state in the sense that the steady state is realized at various epochs and solve the simultaneous equations at the equi-interval mesh-points between the radii of the inner and outer cores by using a Newton method. We just approximate the scalar functions using the values at all the mesh-points. We, however, were unable to find any steady solution. As implied by non-linear equations for a dynamo problem, complicated time variation of the Earth's magnetic field arising from its non-linear nature would be an essential nature of the geodynamo. As the next step, we loose the restriction of the steady state; that is, we solve the simultaneous equations so as to minimize time variations of the velocity and the magnetic fields.

In actual calculations, we treat the equations in dimensionless form scaled in terms of typical strength of magnetic field $B_{s}$, typical magnitude of velocity field $V_{s}$, typical length $L_{s}$, and typical time $T_{s}$. In this paper, we adopt $B_{s}=10^{-3} \mathrm{~T}, V_{s}=10^{-4} \mathrm{~m} / \mathrm{s}, L_{s}=3.485 \times 10^{6} \mathrm{~m}$ (outer core radius), and $T_{s}=L_{s} / V_{s}=3.485 \times 10^{10} \mathrm{~s} \approx 1000$ years. We use the same values of physical constants and properties of the Earth and the core as adopted in MATSUSHIMA and HoNkURA (1992): $\mu_{0}=4 \pi \times 10^{-7} \mathrm{H} / \mathrm{m}, r_{e}=6.371 \times 10^{6} \mathrm{~m}, r_{o c}=3.485 \times 10^{6} \mathrm{~m}, r_{i c}=1.217 \times 10^{6} \mathrm{~m}$, $\rho=1.1 \times 10^{4} \mathrm{~kg} / \mathrm{m}^{3}, \Omega=7.29 \times 10^{-5} \mathrm{rad} / \mathrm{s}, \sigma=3 \times 10^{5} \mathrm{~S} / \mathrm{m}$, and $\nu=1 \mathrm{~m}^{2} / \mathrm{s}$.

It should be noted here that it is impossible to solve non-linear simultaneous equations in a unique manner. In this paper, we selected the Gauss-Newton method, which is based on linear approximation in the Taylor expansion, among three types of algorithm for non-linear least-square method and applied it iteratively. Although damping parameters are usually included to stabilize normal equations, they are not used explicitly in this study. However, the diffusion terms, which tend to smooth the velocity and the magnetic fields, are included in the equations to be solved. It is therefore possible to regard the diffusion coefficients, which have physical meanings, as implicit 
(a)
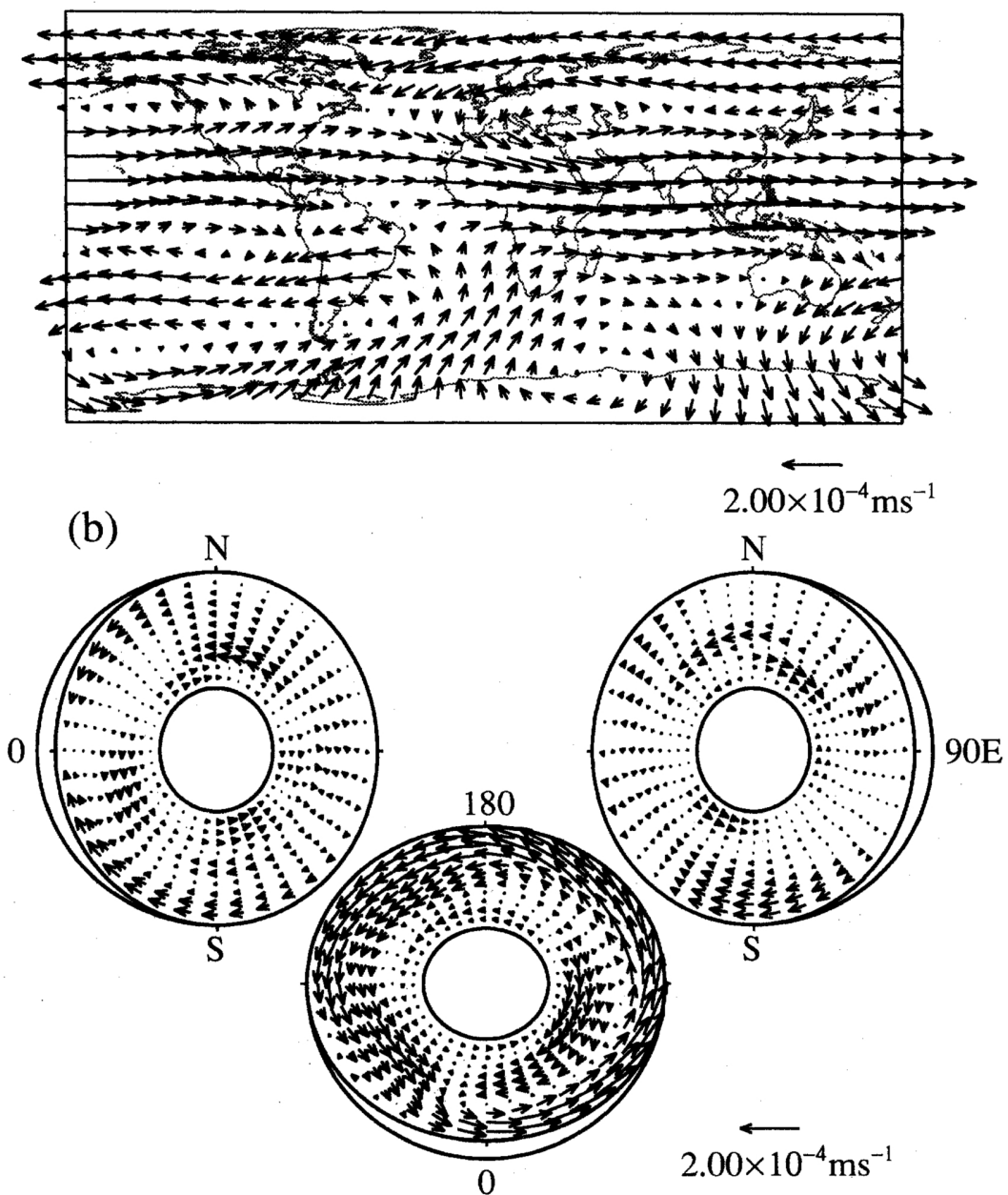

Fig. 3. (a) Horizontal velocity field at the CMB and (b) the overall velocity fields on the equatorial plane and on the meridional planes for the epoch of $1980 \mathrm{AD}$ for $\sigma_{i c}=0$. The arrow for scale length corresponds to $2 \times 10^{-4} \mathrm{~m} / \mathrm{s}$.

damping parameters. In any case, the results given in the next section are considered to be one of the solutions which fit the magnetic field distribution at the CMB and satisfy the imposed conditions; the velocity and the magnetic fields whose time variations are minimum among the obtained solutions.

\section{Results}

In solving the Navier-Stokes and the induction equations, the truncation levels $L$ and $N$ were set at 4 and 10, respectively, as in MAtsushima and HonkURA (1992). For the two extreme values of the electrical conductivity of the inner core, $\sigma_{i c}=0$ and $\sigma_{i c}=\infty$, the derived velocity fields are shown in Figs. 3 and 4, respectively; (a) at the CMB and (b) on the equatorial and on two meridional planes. Non-zonal toroidal motions in Figs. 3 and 4 are clearly dominant, com- 
(a)
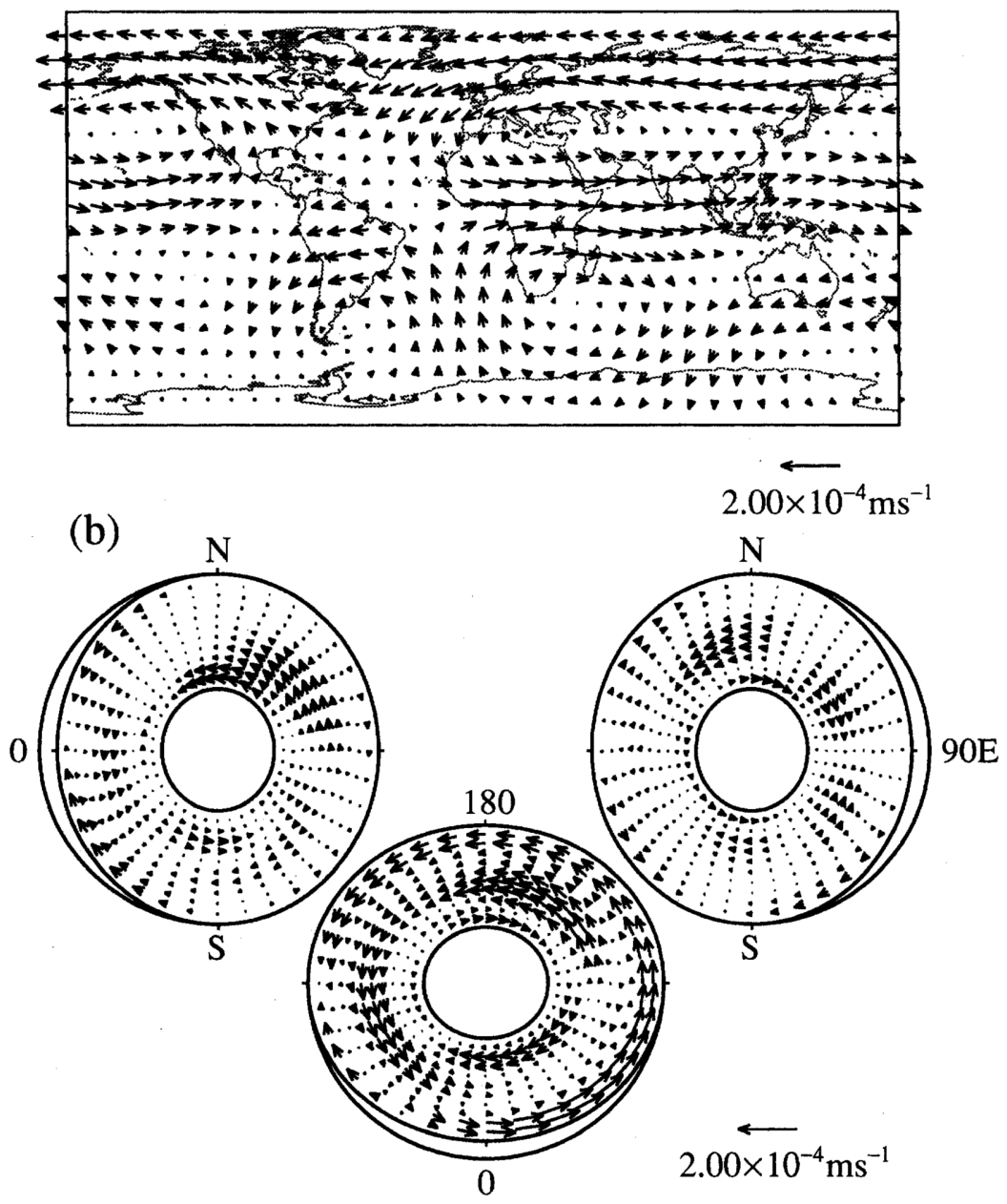

Fig. 4. (a) Horizontal velocity field at the CMB and (b) the overall velocity fields on the equatorial plane and on the meridional planes at the epoch of $1980 \mathrm{AD}$ for $\sigma_{i c}=\infty$. The arrow for scale length corresponds to $2 \times 10^{-4} \mathrm{~m} / \mathrm{s}$.

pared with our previous results (MATSUSHIMA and HONKURA, 1992), in which non-axisymmetric poloidal velocity fields were dominant. The difference comes from the presumption; MATSUSHIMA and HONKURA (1992) presumed a strong $\omega$-effect and assumed that non-axisymmetric poloidal magnetic fields are generated only by the interaction between non-axisymmetric poloidal motions and strong zonal toroidal magnetic fields, whereas all the interaction terms have been taken into account in this paper.

The flow pattern in Fig. 3 is characterized by counterclockwise flows at around $60^{\circ} \mathrm{W}$ in the Southern Hemisphere and at around $120^{\circ} \mathrm{E}$ in the Northern Hemisphere, and by westward flow near the North Pole, while that in Fig. 4 is by a clockwise flow at around $20^{\circ} \mathrm{E}$ in the Southern Hemisphere and counterclockwise flows at around $140^{\circ} \mathrm{W}$ in the Northern and Southern Hemispheres. The present approach is entirely different from that based on the frozen-flux approximation, and it is therefore not surprising that the fluid motions shown in Figs. 3 and 4 do 
(a)
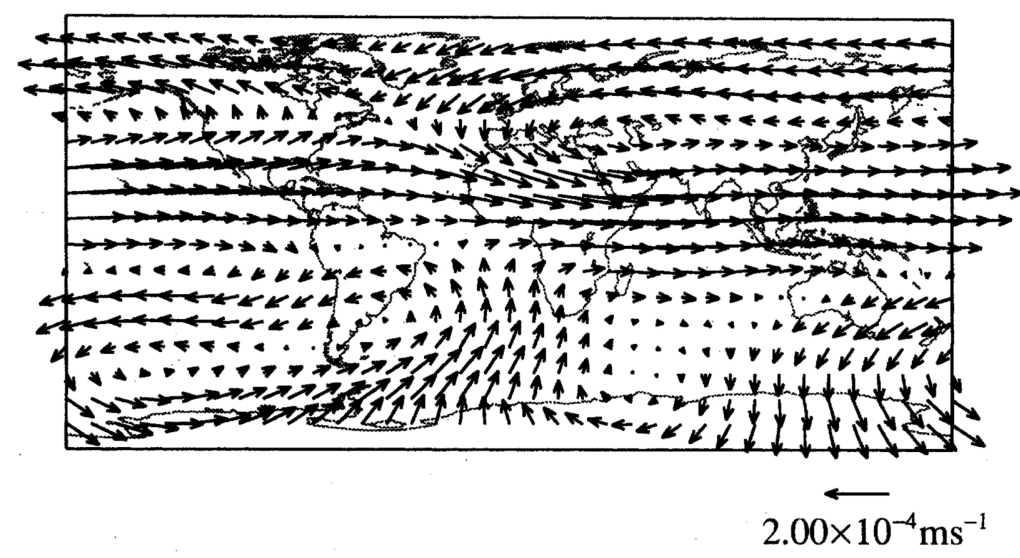

(b)

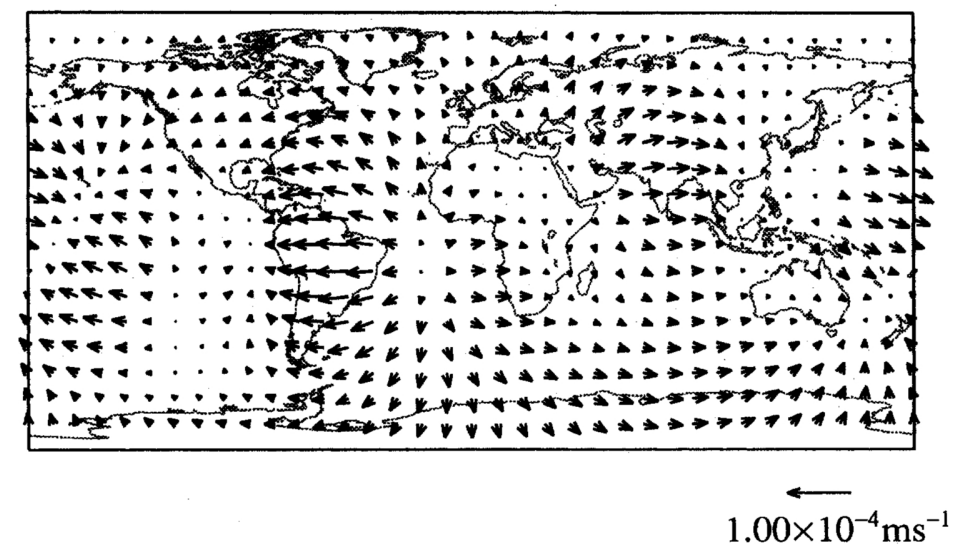

Fig. 5. (a) The toroidal constituent and (b) the poloidal constituent of the velocity field shown in Fig. 3 . The arrow for scale length in (b) is different from that in (a).

not coincide with those derived with the frozen-flux approximation by LE MouËL et al. (1985), Voorhies (1986), Whaler and Clarke (1988), Bloxham (1989), and Lloyd and Gubbins (1990).

The toroidal and poloidal constituents of the derived velocity field for $\sigma_{i c}=0$ (Fig. 3) are shown in Figs. 5(a) and 5(b), respectively. It should be noticed that the scale length for arrows in Fig. 5(b) is different from that in Fig. 5(a). It is clearly recognized that the toroidal velocity field at the CMB is dominant. If we compare the magnitude of toroidal and poloidal constituents in terms of the root-mean-square velocity, which is defined by

$$
\langle V\rangle=\left(\frac{\int \boldsymbol{V}^{2} \mathrm{~d} v}{\int \mathrm{d} v}\right)^{1 / 2},
$$

where the volume integral is performed for the outer core, then we have $\left\langle V_{T}\right\rangle=8.37 \times 10^{-5} \mathrm{~m} / \mathrm{s}$ 
and $\left\langle V_{S}\right\rangle=1.66 \times 10^{-5} \mathrm{~m} / \mathrm{s}$. The toroidal velocity field is therefore dominant throughout the outer core by a factor of 5 . A similar result is obtained for the velocity field derived for $\sigma_{i c}=\infty$, shown in Fig. 4: $\left\langle V_{T}\right\rangle=4.90 \times 10^{-5} \mathrm{~m} / \mathrm{s}$ and $\left\langle V_{S}\right\rangle=1.06 \times 10^{-5} \mathrm{~m} / \mathrm{s}$. The toroidal velocity field is dominant by a factor of 4.5. These factor values are consistent with the factor of four for the toroidal component to the consoidal one of tangentially geostrophic flow at the core surface, derived on the frozen-flux approximation by GIRE and LE MOUËL (1990).

\section{Discussion}

\subsection{Time variations}

We solved the Navier-Stokes and the induction equations so as to minimize time variations of the velocity and the magnetic fields. In order to show to what extent time variations are small, we calculate the time variation of the magnetic field using the derived velocity field and compare it with the observed one. Figure 6 shows the distributions of the rate of time variation for the

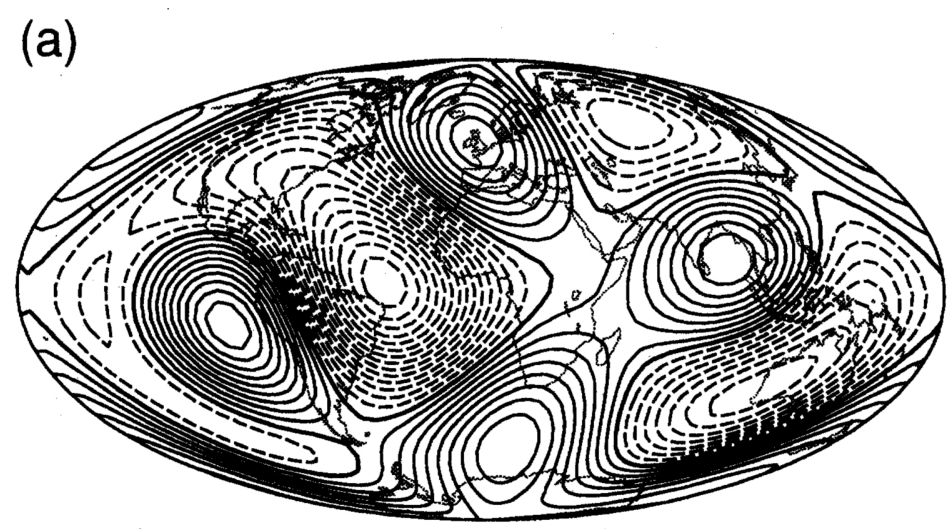

(b)

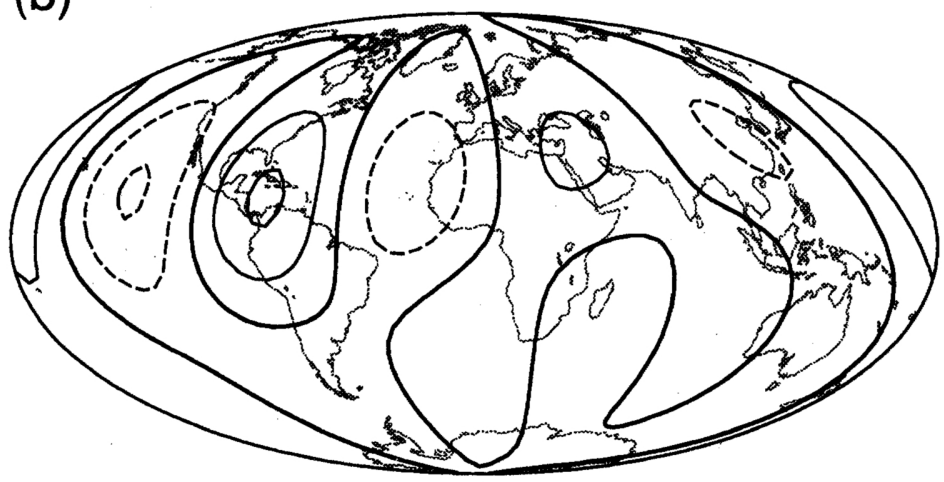

Fig. 6. Distributions of the rate of time variations for the radial component of the magnetic field at the CMB up to degree four for the epoch of 1980 AD; (a) derived from the DGRF's and (b) calculated using the velocity field shown in Fig. 3 for $\sigma_{i c}=0$. Solid contours represent positive variations and broken contours negative variations, in units of $0.2 \mu \mathrm{T} /$ year. 
radial component of the magnetic field $\left(\dot{B}_{r}\right)$ at the CMB up to degree four for the epoch of 1980 $\mathrm{AD}$; (a) derived from the DGRF's and (b) calculated using the velocity field shown in Fig. 3 for $\sigma_{i c}=0$. Contour intervals are $0.2 \mu \mathrm{T} /$ year. It turns out from the $\dot{B}_{r}$ distributions that the derived magnetic field is in the nearly steady state for the derived velocity field.

The actual Earth's magnetic field varies with time, and one may ask what meaning the derived models have. The present models would be compared with models derived on the basis of the frozen-flux approximation (RoBERTS and SCOTT, 1965; BLOXHAM and JACKSON, 1991), in which the time variation and the radial component of poloidal magnetic field at the CMB are used to estimate core surface flow. The motivation for these studies is an expectation that the derived models might give information on the dynamo process within the core. The distinction between those in the frozen-flux approximation and the present models is whether or not the model can be a self-exciting dynamo model; that is, the magnetic field as observed at the Earth's surface is generated by the induction process and maintained against Ohmic dissipation in the model, although the observed secular variation is incompatible with the present models.

In order to derive more realistic fluid motion in the core, we must take time variations (secular variations) into consideration. Since time variation of the poloidal magnetic field only can be observed at the Earth's surface, this is an extremely difficult problem. Moreover, even for the poloidal magnetic field, time variations within the core are unknown. The frozen-flux approximation may be instructive in a sense. At the present time, in any case, the problem remains to be solved in the future.

\subsection{Electrical conductivity of the inner core}

We considered two extreme cases of the electrical conductivity of the inner core $\left(\sigma_{i c}=0\right.$ and $\left.\sigma_{i c}=\infty\right)$ in deriving fluid motion within the core. The patterns of fluid motion at the core surface turn out to be similar to each other (Figs. 3 and 4) to some extent. However, there would be differences near the ICB because of the different boundary conditions for the magnetic field there. Figure 7 shows the magnetic lines of force for axisymmetric poloidal fields (left semicircle) and the distribution of strength of zonal toroidal magnetic fields (right semicircle) for the two cases; (a) for $\sigma_{i c}=0$ and (b) for $\sigma_{i c}=\infty$.

It is found that the magnetic lines of force for axisymmetric poloidal fields in Fig. 7(a) are
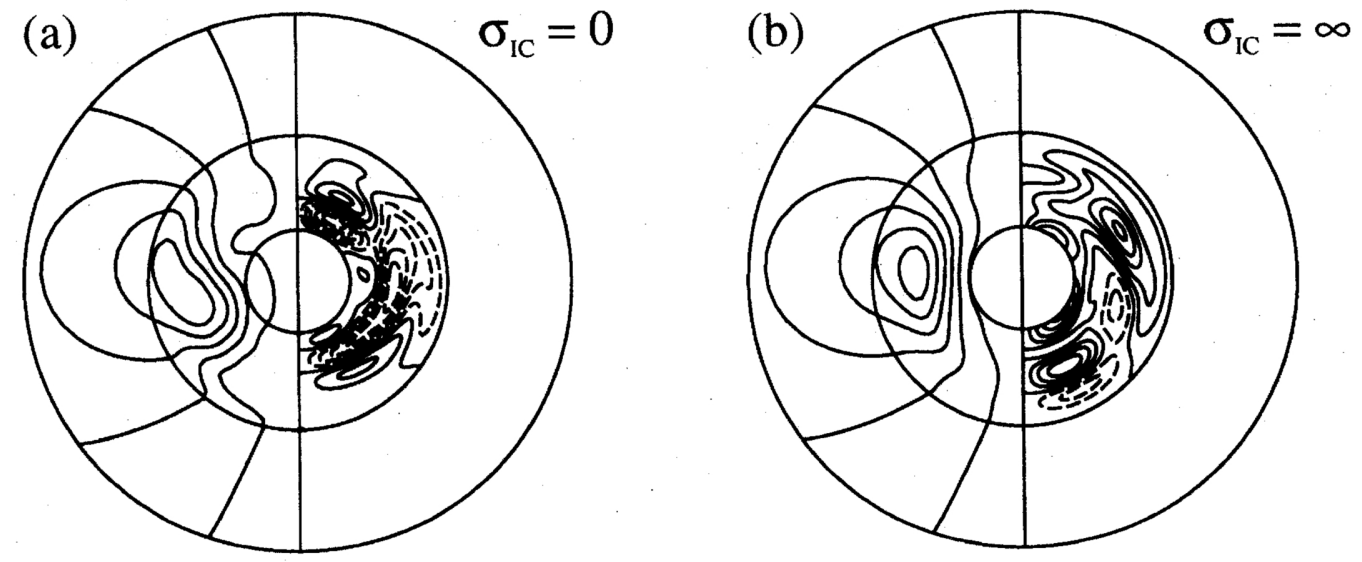

Fig. 7. Magnetic lines of force for axisymmetric poloidal fields (left semicircle) and the distributions of strength of zonal toroidal magnetic fields (right semicircle); (a) for $\sigma_{i c}=0$ and (b) for $\sigma_{i c}=\infty$. Contour intervals for zonal toroidal magnetic fields are $4 \times 10^{-4} \mathrm{~T}$. 
similar to those in Fig. 7(b). This would result from the fact that the volume of the inner core is very small, as suggested by ZHANG and Busse $(1988,1989)$. On the other hand, the distribution of strength of zonal toroidal magnetic fields in Fig. 7(a) is different from that in Fig. 7(b); the toroidal magnetic field can be strong near the ICB, because of $\sigma_{i c}=\infty$. We should treat the electrical conductivity of the inner core more carefully.
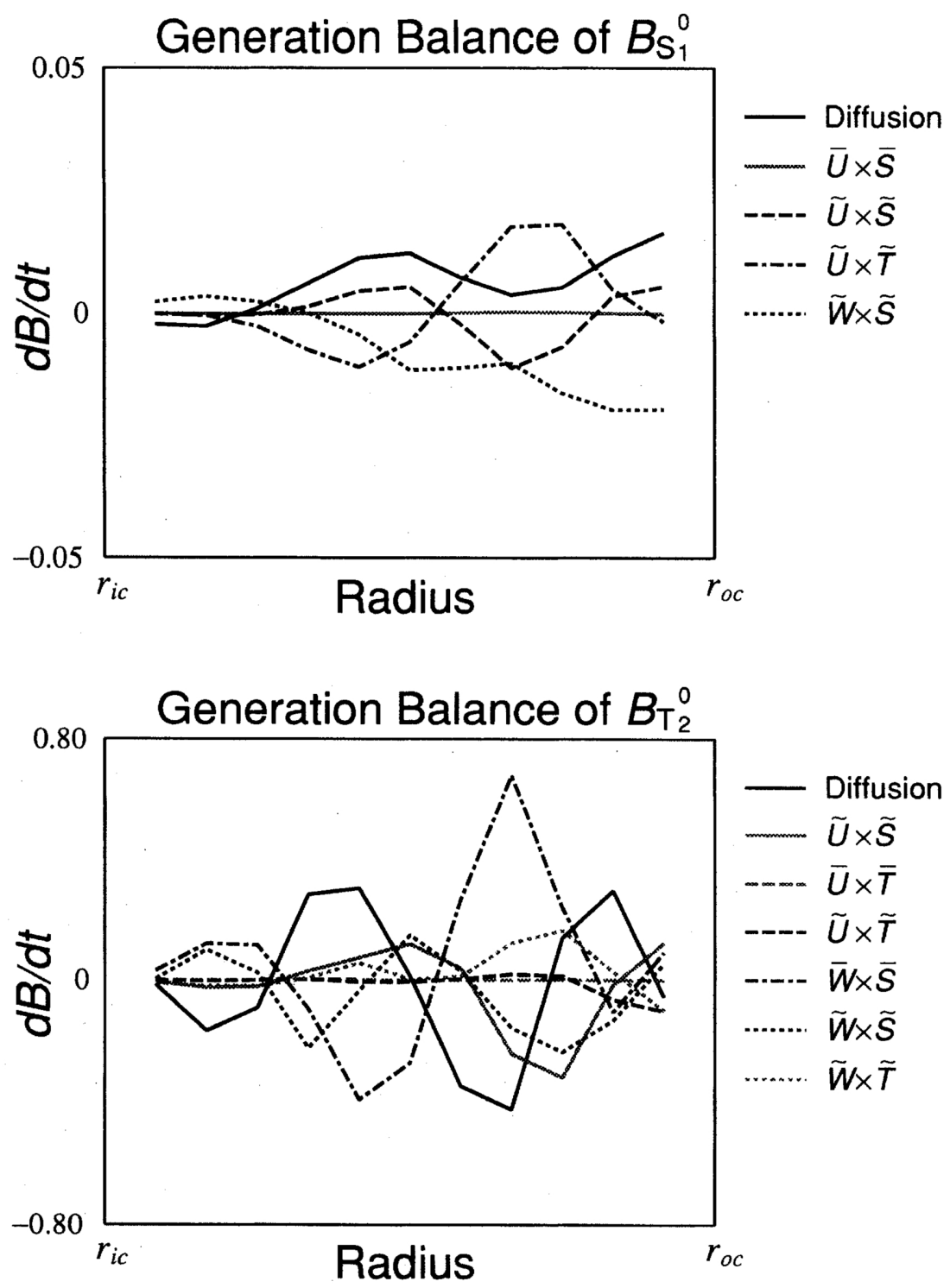

Fig. 8. Generation balance of $S_{1}^{0}$ (upper) and $T_{2}^{0}$ (lower) in the induction equation for $\sigma_{i c}=0$. Shown are radial dependence of non-dimensional values of $\partial S_{1}^{0} / \partial t$ and $\partial T_{2}^{0} / \partial t$ between $r_{i c}$ and $r_{o c}$ for the diffusion and the induction terms, where $U \times S$, for example, denotes the interaction between poloidal velocity and poloidal magnetic fields, and bar and tilde express the axisymmetric and the non-axisymmetric components, respectively. 


\subsection{Whole core convection}

We have presumed whole core convection and prescribed the radial dependence of poloidal velocity field, accordingly. Whole core convection is suggested by JACKSON and BLOXHAM (1991) who derived fluid motion and the shear, based on the frozen-flux approximation, near the core surface using the radial and horizontal components of the magnetic field. They found that the shear near the core surface is weak and aligned with the flow; that is, the flow is highly correlated with the flow deep within the core. On the other hand, LLOYD and GuBBINs (1990) suggest that fluid flow is confined to the $600 \mathrm{~km}$ depth near the core surface. The difference of their results depends on a damping parameter in solving equations.

One way to clarify the type of convection would be to study the energy source for fluid flow within the core. LLOYD and GUBBINS (1990) explain that the fluid flow in a stratified layer near the core surface would be driven by lateral temperature variations in the lower mantle, as suggested by BLOXHAM and GuBBINS (1987). On the other hand, whole core convection could be driven not only by internal origin, for example, light elements released in association with the growth of the inner core or thermal energy, but also by lateral temperature variations in the lower mantle (ZHANG and GuBBINS, 1992). In any case, further investigation would be required.

We have attempted to use several other functional forms for the radial dependence of poloidal velocity field which express one-layer whole core convective motions ${ }^{\dagger}$. The derived velocity fields are not significantly different. Therefore, as long as we assume such convective motions, the conclusion which will be described in the next subsection would hold.

\subsection{Geodynamo mechanism}

In order to examine which type of dynamo mechanism is relevant to the geodynamo, we consider the generation balance in the induction equation. Dominant modes in $\alpha \omega$-dynamos have traditionally been considered as the axial dipole magnetic field $\boldsymbol{B}_{S_{1}}^{0}$ and the zonal toroidal magnetic field $\boldsymbol{B}_{T 2}^{0}$, which is expected to be generated by differential rotation. We therefore evaluate generation terms on the right-hand-side of Eqs. (6) and (7) for $T_{2}^{0}$ and $S_{1}^{0}$, respectively. The contributions from each term for $\sigma_{i c}=0$ are shown in Fig. 8, where $U \times S$, for example, represents the generation by the interaction between poloidal velocity and poloidal magnetic fields; that is, $\left(U_{\alpha} S_{\beta} s_{\gamma}\right)$ or $\left(U_{\alpha} S_{\beta} t_{\gamma}\right)$. The bar and tilde in Fig. 8 denote axisymmetric and non-axisymmetric components, respectively.

It is seen from Fig. 8 that the generation of $S_{1}^{0}$ is mainly due to the interaction between non-axisymmetric velocity and non-axisymmetric magnetic fields. On the other hand, $\bar{W} \times$ $\bar{S}$, which corresponds to the $\omega$-effect, also contributes to the generation of $T_{2}^{0}$ in addition to the interaction between non-axisymmetric velocity and non-axisymmetric magnetic fields. In typical $\alpha \omega$-dynamos, the generation of zonal toroidal magnetic fields by the $\omega$-effect is much more dominant than by the $\alpha$-effect. As seen in Fig. 8, however, the $\alpha$-effect also contributes to the generation of $T_{2}^{0}$. In this sense, it is concluded that the geodynamo is likely to be of $\alpha^{2} \omega$-type.

\section{Conclusions}

We derived fluid motion in the Earth's outer core from geomagnetic field data by solving both the induction and the Navier-Stokes equations so as to minimize time variations of the velocity and the magnetic fields. We prescribed the radial dependence of poloidal velocity field and considered two extreme values of the electrical conductivity of the inner core. We did not rely on any approximations for dominant induction terms, such as the $\omega$-effect, as in MATSUSHIMA and HoNKURA (1992), but took all the interaction terms into consideration. The results are summarized as follows.

\footnotetext{
${ }^{\dagger}$ In order to express one-layer convection, ${ }_{1} U_{l}^{m}$ given in (15) must be dominant.
} 
(1) For the two values of the electrical conductivity of the inner core, $\sigma_{i c}=0$ and $\sigma_{i c}=\infty$, there is little difference between the derived fluid motions within the core as far as (2) and (3) below are concerned. It is pointed out, however, that the toroidal magnetic field can be strong near the ICB for a large value of $\sigma_{i c}$.

(2) The toroidal velocity field is more dominant than the poloidal velocity field as in core surface motions derived on the frozen-flux approximation. The result is also applied to fluid motion within the core.

(3) The generation balance for the axial dipole magnetic field $\boldsymbol{B}_{S_{1}^{0}}^{0}$ and the zonal toroidal magnetic field $\boldsymbol{B}_{T 2}^{0}$ was examined in the induction equation and it was found that both the $\alpha$ and the $\omega$-effects contribute to the generation of $T_{2}^{0}$. This result suggests that the geodynamo is likely to be of $\alpha^{2} \omega$-type.

We have assumed whole core convection. The validity of this assumption would be verified by clarifying the energy source for dynamo action. In order to understand a more realistic dynamo mechanism, we must take into consideration secular variations of the Earth's magnetic field in deriving fluid motion within the core.

The author thanks Dr. G. Hulot and another anonymous referee for their useful comments on the manuscript. The author would also like to express his sincere thanks to Dr. Y. Honkura for his valuable discussion and reading this paper. The author was supported by the Japan Society for the Promotion of Science as a fellow for Japanese Junior Scientists. This work was partially supported by the Ministry of Science, Education and Culture, Japan. This paper was presented at the 3rd SEDI (Study of the Earth's Deep Interior) symposium held at Mizusawa, Japan, in 1992. The calculation was performed on ETA ${ }^{10}$ at the Computer Center in the Tokyo Institute of Technology. Contribution of the Department of Earth and Planetary Sciences, Tokyo Institute of Technology, No. 12.

\section{REFERENCES}

BAckUs, G. E., Kinematics of the geomagnetic secular variation in a prefectly conducting core, Phil. Trans. Roy. Soc. Lond. A, 263, 239-266, 1968.

Bloхнам, J., Simple models of fluid flow at the core surface derived from geomagnetic field models, Geophys. J. Int., 99, 173-182, 1989.

Bloxham, J. and D. GubBins, Thermal core-mantle interactions, Nature, 325, 511-513, 1987.

Bloxham, J. and A. JACKSON, Fluid flow near the surface of Earth's outer core, Rev. Geophys., 29, 97-120, 1991.

Bullard, E. C. and H. Gellman, Homogeneous dynamos and terrestrial magnetism, Phil. Trans. Roy. Soc. Lond. $A$, 247, 213-278, 1954.

Frazer, M. C., Spherical harmonic analysis of the Navier-Stokes equation in magnetofluid dynamics, Phys. Earth Planet. Inter., 8, 75-82, 1974.

GIRE, C. and J.-L. LE MOUËL, Tangentially geostrophic flow at the core-mantle boundary compatible with the observed geomagnetic secular variation: the large-scale component of the flow, Phys. Earth Planet. Inter., 59, 259-287, 1990.

Glatzmaier, G. A., Numerical simulations of stellar convective dynamos, 1. The model and method, J. Comp. Phys., 55, 461-484, 1984.

Gubbins, D., Finding core motions from magnetic observations, Phil. Trans. Roy. Soc. Lond. A, 306, 247-254, 1982.

Honkura, Y. and M. Matsushima, Time-dependent pattern of core motion inferred from fluctuations of standing and drifting non-dipole fields, J. Geomag. Geoelectr., 40, 1511-1522, 1988.

Honkura, Y. and T. Rikitake, Core motion as inferred from drifting and standing non-dipole fields, J. Geomag. Geoelectr., 24, 223-230, 1972.

IAGA Division I Working Group 1, International Geomagnetic Reference Field Revision 1987, Geophys. J., 93, 187-189, 1988.

JACKSON, A. and J. Bloxham, Mapping the fluid flow and shear near the core surface using the radial and horizontal components of the magnetic field, Geophys. J. Int., 105, 199-122, 1991.

LE Moü̈L, J.-L., C. GIRE, and T. MADDEN, Motions at core surface in the geostrophic approximation, Phys. Earth Planet. Inter., 39, 270-287, 1985.

Lloyd, D. and D. GubBins, Toroidal motion at the top of the Earth's core, Geophys. J. Int., 100, 455-467, 1990.

Matsushima, M. and Y. HonkuRA, Large-scale fluid motion in the Earth's outer core estimated from non-dipole magnetic field data, J. Geomag. Geoelectr., 41, 963-1000, 1989. 
Matsushima, M. and Y. HonkuRA, Reexamination of fluid motion in the Earth's core derived from geomagnetic field data-Is the $\omega$-effect really strong in the core?, J. Geomag. Geoelectr., 44, 521-553, 1992.

RIKITAKE, T., Non-dipole field and fluid motion in the earth's core, J. Geomag. Geoelectr., 19, $129-142,1967$.

Roberts, P. H. and S. ScotT, On analysis of the secular variation, 1. A hydromagnetic constraint: theory, $J$. Geomag. Geoelectr., 17, 137-151, 1965

Voorhies, C. V., Steady flows at the top of earth's core derived from geomagnetic field models, J. Geophys. Res., 91, 12,444-12,466, 1986.

Whaler, K. A., Does the whole of the Earth's core convect?, Nature, 287, 528-530, 1980.

Whaler, K. A. and S. O. Clarke, A steady velocity field at the top of the Earth's core in the frozen-flux approximation, Geophys. J., 94, 143-155, 1988.

ZHANG, K.-K. and F. H. Busse, Finite amplitude convection and magnetic field generation in a rotating spherical shell, Geophys. Astrophys. Fluid Dyn., 44, 33-53, 1988.

ZhANG, K.-K. and F. H. Busse, Convection driven magnetohydrodynamic dynamos in rotating spherical shells, Geophys. Astrophys. Fluid Dyn., 49, 97-116, 1989.

ZhANG, K. and D. GubBINS, On convection in the earth's core driven by lateral temperature variations in the lower mantle, Geophys. J. Int., 108, 247-255, 1992. 